\title{
EMC-oriented Multi-conductor Equivalent Circuit Cable Models for Spice, including Transfer Impedance Coupling and Incident Field Excitation
}

\author{
C. Smartt, M.J. Basford, S. Greedy, D.W.P. Thomas and M. Sumner
}

\begin{abstract}
This paper describes the development and application of sophisticated multi-conductor cable bundle models for Spice simulation. The cable models developed include frequency dependent parameters, transfer impedance coupling and incident plane wave excitation. The models may be used in frequency domain or transient simulations and are intended for EMC/ EMI studies. Simulations of realistic scenarios are presented to demonstrate typical applications.
\end{abstract}

\section{INTRODUCTION}

Along with developments in electronic systems such as higher speed digital lines, lower voltage digital signals, Power Line Communication (PLC) and Power over Ethernet (PoE) for example, comes an increased reliance on the performance of the cables and associated connectors used in system interconnects. It is therefore highly desirable to include the effects of complex cable bundles into the simulation of the system as a whole, including the effects of crosstalk on multi-conductor cable bundles, transfer impedance coupling and incident field excitation, whilst including frequency dependent effects such as skin effect losses.

In this paper we demonstrate how accurate models of complex multi-conductor cable systems can be constructed from simple circuit elements (lumped impedances, controlled sources, delay lines and sdomain transfer functions) which include all the desired propagation and coupling effects. These models have been developed for implementation as Spice sub-circuits which may be embedded within circuit models and used in both frequency domain and time domain simulations.

Software which generates these Spice cable bundle models has been developed in the open source project 'SACAMOS' [1]. This toolset provides EMC-oriented cable models which are readily and freely available for use in the circuit simulation tool Spice.

\section{Spice multi-conductor transmission line model description}

There has been considerable effort over many years in the development of Spice models for complex transmission line systems including multi-mode propagation [2], shielded cables [3] and incident field excitation [4]. Most of these models have been derived for idealized (lossless) transmission lines, and often for very specific configurations. In this section we describe how these methods may be corrected for more realistic transmission lines with (frequency dependent) losses included.

The voltage and current on a transmission line satisfy the coupled equations [2]

$$
\begin{aligned}
& \frac{\partial}{\partial z}(V(z, t))=-[R] I(z, t)-[L] \frac{\partial}{\partial t} I(z, t) \\
& \frac{\partial}{\partial z}(I(z, t))=-[G] V(z, t)-[C] \frac{\partial}{\partial t} V(z, t)
\end{aligned}
$$

In the frequency domain we may write

$$
\begin{aligned}
& \frac{d}{d z}(\tilde{V}(z))=-[\tilde{Z}] \tilde{I}(z) \\
& \frac{d}{d z}(\tilde{I}(z))=-[\tilde{Y}] \tilde{V}(z)
\end{aligned}
$$

Where

$$
\begin{aligned}
& {[\tilde{Z}]=[R]+j \omega[L]} \\
& {[\tilde{Y}]=[G]+j \omega[C]}
\end{aligned}
$$

The solution of transmission line systems is frequently sought using the technique of modal decomposition where we may start from one of the uncoupled second order equations

$$
\frac{d^{2}}{d z}(\tilde{V}(z))=[\tilde{Z}][\tilde{Y}](\tilde{V}(z)) \quad \frac{d^{2}}{d z}(\tilde{I}(z))=[\tilde{Y}][\tilde{Z}](\tilde{I}(z))_{(0.4)}
$$

The modal decomposition is based on a change of variables $(\tilde{V}(z))=\left[\tilde{T}_{V}\right]\left(\tilde{V}_{m}(z)\right)$ which diagonalises the matrix system e.g. for the voltage based equation 1.5 we have

$$
\frac{d^{2}}{d z}\left(\tilde{V}_{m}(z)\right)=\left[\tilde{T}_{V}\right]^{-1}[\tilde{Z}][\tilde{Y}]\left[\tilde{T}_{V}\right]\left(\tilde{V}_{m}(z)\right)=\left[\gamma_{0}^{2}\right]\left(\tilde{V}_{m}(z)\right)
$$

The solution to this equation may be expressed in terms of voltage waves in the $\pm \mathrm{z}$ directions on the transmission line i.e. the method of characteristics [2]

$$
\tilde{V}_{m}(z)=\tilde{V}^{+} e^{-\gamma_{m} z}+\tilde{V}^{-} e^{+\gamma_{m} z} \quad \tilde{I}_{m}(z)=\frac{1}{Z_{0 m}}\left(\tilde{V}^{+} e^{-\gamma_{m} z}-\tilde{V}^{-} e^{+\gamma_{m} z}\right)
$$

A Spice model which implements the method of characteristics for simple, lossless, frequency independent transmission lines, including for multiconductor systems is described in [2]. This model has also been developed so as to include the effect of incident plane wave excitation. The incident plane wave induces distributed sources on the conductors [5]. The effect of these sources on the terminations may be included in the Spice circuit model using lumped sources at the transmission line terminations.

Spice models for shielded cables, including transfer impedance coupling through imperfect shields have 
been derived for a coaxial cable above a ground plane by Xie et al [3]. This model is based on the method of characteristics and develops Paul's incident field excitation model [4] to include the weak form of transfer impedance coupling (i.e. a one way coupling model). In this work we have extended this model to include multi-mode propagation and the effects of frequency dependent loss mechanisms.

One of the main difficulties in deriving simulation models for realistic transmission lines, especially in the time domain, is the incorporation of losses which can include frequency dependent effects. These may arise from the finite conductivity of the conductors, which leads to a frequency dependent resistance (skin effect) and inductance, and the effect of frequency dependent dielectrics used in the cable construction. The effect on the analysis of transmission lines is that the propagation constant, $\gamma=\sqrt{(R+j \omega L)(G+j \omega C)}$, wave impedance, $Z_{0}=\frac{\sqrt{R+j \omega L}}{\sqrt{G+j \omega C}}$ and modal decomposition matrix, $\left[\tilde{T}_{V}\right]$, become a function of frequency.

If we are interested in simulating cable lengths of the order of a few meters, as would be found in many systems (automotive, satellite systems etc), over a frequency range from d.c. to $1 \mathrm{GHz}$ then a viable model may be derived which avoids these difficulties by making the following assumptions

1. We may lump the d.c. resistance of conductors at the terminations of transmission lines

2. We assume that the d.c. admittance is zero

3. The modal decomposition matrices may be derived for a lossless transmission line using 'high frequency' values of inductance and capacitance

The first assumption ensures that the model will be correct at d.c. The third assumption is justified in that the $[\tilde{Z}][\tilde{Y}]$ product is dominated by the $-\omega^{2} L C$ term. The transmission line losses may be corrected for dispersion and loss by multiplying the waves on the transmission line by a 'propagation' correction. This propagation correction is frequency dependent and defined as $H(j \omega)=e^{-\left(\gamma^{\prime}(j \omega)-\gamma_{0}\right) L} \quad$ where $\gamma^{\prime}(j \omega)=\sqrt{R(j \omega)-R_{d c}+j \omega L(j \omega)} \sqrt{G(j \omega)+j \omega C(j \omega)}$ and $\gamma_{0}=\sqrt{-\omega^{2} L_{0} C_{0}}$ are the propagation constants for the frequency dependent transmission line and the idealised, lossless transmission line respectively. It is important to note that the d.c. resistance is subtracted as this is included in the model at the conductor termination, also, even though we assume that $\mathrm{G}=0$ at d.c. this does not preclude the existence of a frequency dependent admittance originating from frequency dependent dielectrics in the cable. This model has been demonstrated to be very accurate over a wide frequency range and for a wide range of termination impedances [6] and generalises to multi-mode propagation. In practice the propagation correction is split between the two ends of the transmission line, half the correction is applied when launching the characteristic voltage waves from the terminations and the other half when they are received at the other end of the transmission line. The frequency dependent transfer function is implemented in Spice using the s-domain transfer function circuit element which may be operated in both time and frequency domain models.

The model can be thought of as an ideal transmission line with a termination network which adds in the effects of the imperfect propagation. This is similar in concept to the Zobel network in which a termination network is used to cancel the dispersive effects of imperfect transmission lines.

To summarise, the Spice cable bundle model is based on a domain decomposition (for shielded cables) then modal decomposition within each domain and a mode propagation algorithm based on the method of characteristics [2]. The mode propagation algorithm incorporates the 'propagation correction' to correct for frequency dependent dispersion and losses on the transmission lines. Imperfect shields are taken into account through lumped controlled source terms whose value is a function of the current on the shield and the transfer impedance [3]. The effect of a plane wave illumination of a cable bundle may also be included in the model through equivalent lumped source terms whose value is a function of the incident field $\mathrm{k}$ vector, polarisation and cable bundle conductor configuration $[3,4]$.

Models of twisted pairs and shielded twisted pairs are included. These models assume that there is no interaction between the differential mode and any other mode in the configuration, coupling is only to the common mode.

Extensive validation against analytic solutions for a wide range of configurations has been performed.

\section{Electrostatic Discharge model}

As a first demonstration of the Spice cable models, we simulate an electrostatic discharge test. The ESD pulse is generated by a Spice circuit consisting of a $150 \mathrm{pF}$ capacitor with an initial voltage of $12 \mathrm{KV}$ which is discharged through series connected $330 \Omega$ resistance and $2 \mu \mathrm{H}$ inductance into the test point. The test object is a RG58 cable with pigtails at either end as seen in figure 1. The RG58 cable model includes the effect of the finite conductivity of the conductors and a transfer impedance model where the transfer impedance is of the form $\mathrm{Z}_{\mathrm{T}}=\mathrm{R}_{\mathrm{T}}+\mathrm{j} \omega \mathrm{L}_{\mathrm{T}}$. The pigtails are included in the model as short two wire 
transmission lines cascaded with the main cable. The discharge point is where the shield connects to the pigtail. Figure 2 shows the end 2 voltage resulting from the ESD pulse showing the effect of the inductive pigtail and the ringing in the shield-ground plane circuit.

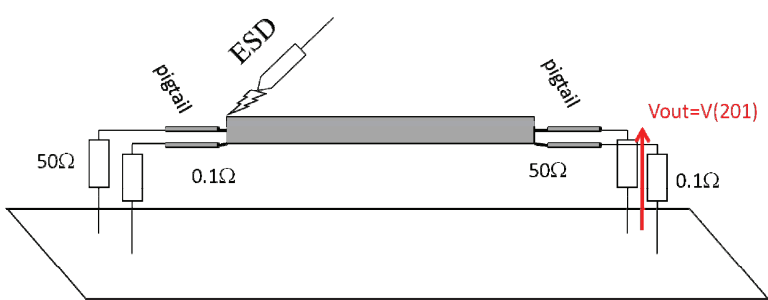

Figure 1. ESD test configuration

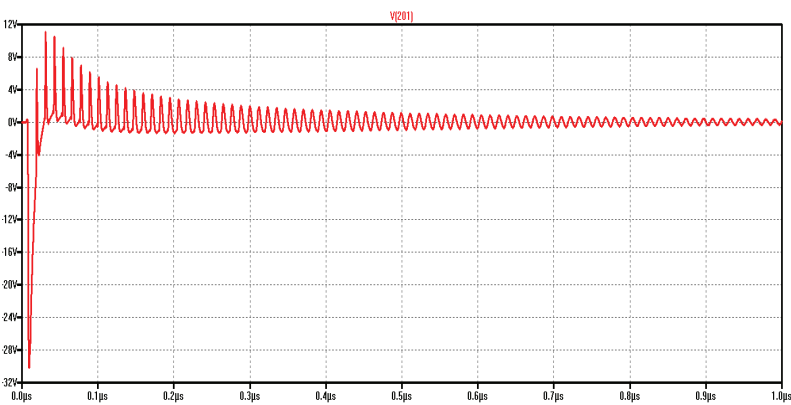

Figure 2. End 2 voltage as a result of the ESD test.

\section{CANBUS network model}

A second application is the simulation of a CANBUS [7] network which incorporates Spice models of driver (figure 3) and receiver (figure 4) circuits on the terminations of a shielded twisted pair transmission line network as seen in figure 5.

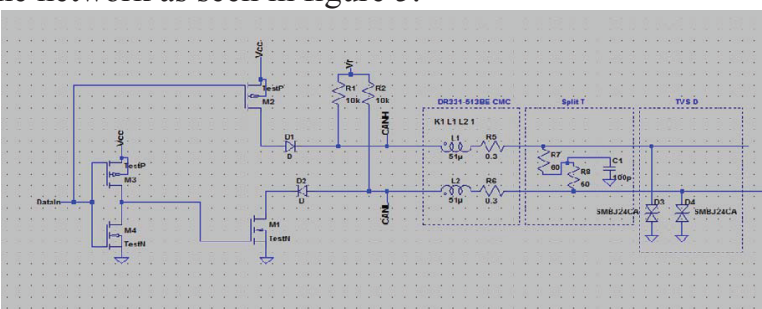

Figure 3. CANBUS transmitter circuit.

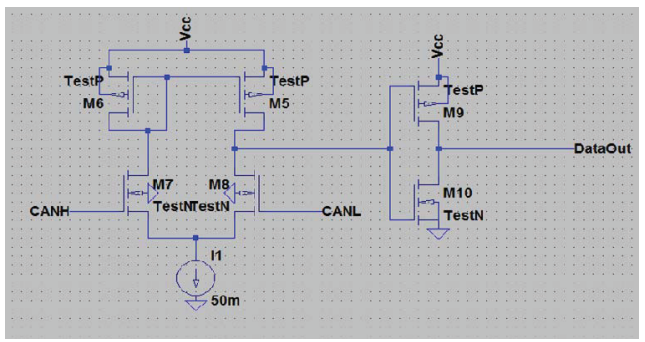

Figure 4. CANBUS receiver circuit

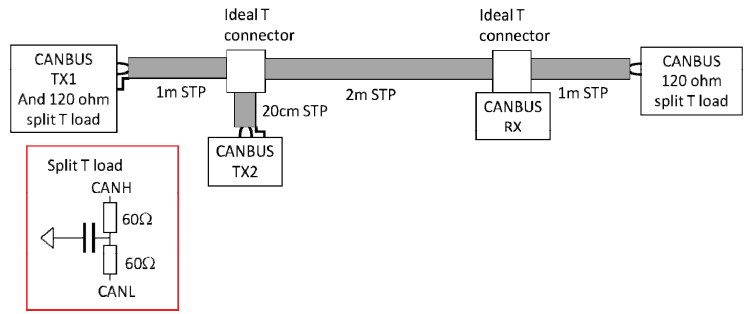

Figure 5. CANBUS network model

Figures 6 and 7 show the voltages on the individual CANBUS conductors and the common mode voltage respectively for a simple transmitter circuit with no common mode choke. Clearly the transmitting circuit is putting significant common mode onto the shielded twisted pair. Figure 8 shows the significant reduction in the common mode voltage of including a common mode choke in the transmitter circuit.

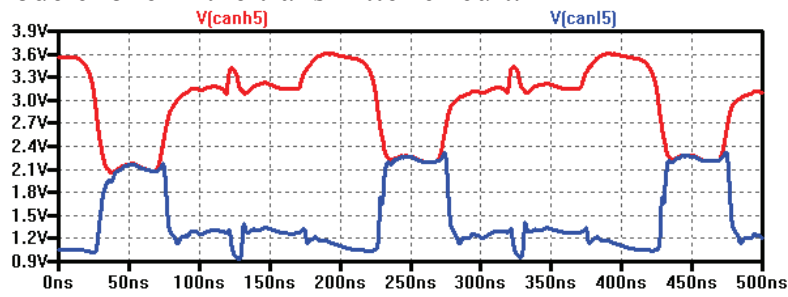

Figure 6. CANH and CANL voltages

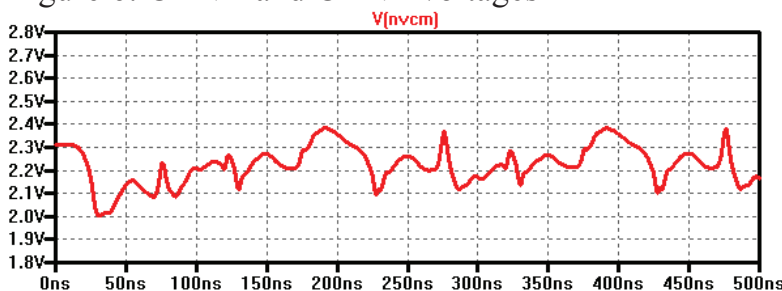

Figure 7. Common mode voltage

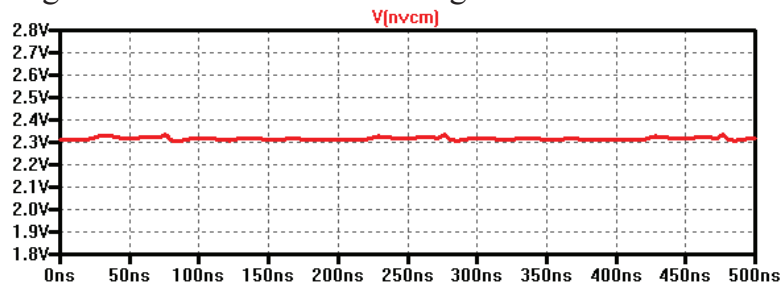

Figure 8. Common mode voltage with common mode choke in the transmitter circuit

\section{Spacewire crosstalk model}

As a final demonstration we study the propagation of signals in a spacewire system [8]. Spacewire consists of four shielded twisted pairs within an over-shield terminated with micro D connectors. The spacewire system uses low voltage differential signaling with a data and a strobe line for communication in each direction. A spacewire model has been constructed which includes the frequency dependent effects of the finite conductivity of the conductors and models of connectors on either end as seen in figure 9. This is cascaded with the micro D connector model in figure 10 . 


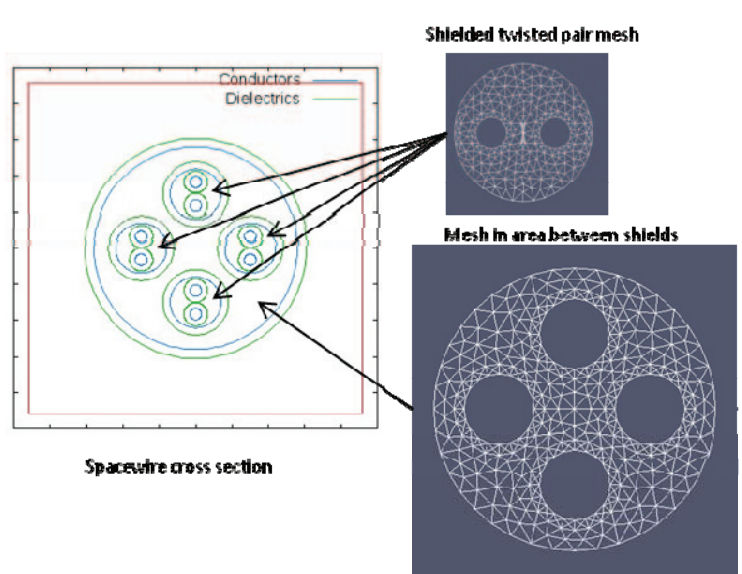

Figure 9. Spacewire cross section and meshes used for calculation of the per-unit-length inductance and capacitance matrices.

D connectar mesh

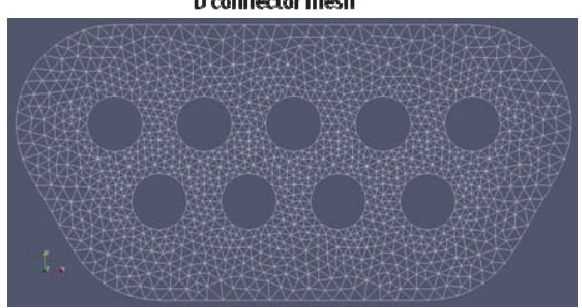

Figure 10. Micro D connector model.

The wiring of the micro D connector is shown in figure 11 showing the transmitting data and strobe lines in both directions and the shield terminations in the connector. The connector allows some crosstalk between the shielded twisted pairs in the model.

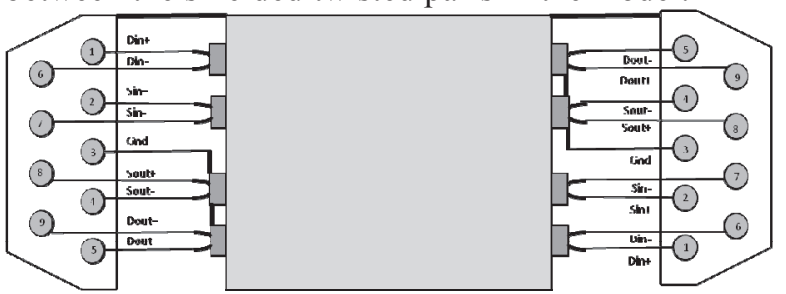

Figure 11. Spacewire model with connectors

Figure 12. Shows the differential mode voltage at the sending end (red) and receiving end (blue) clearly showing the effect of the frequency dependent propagation losses.

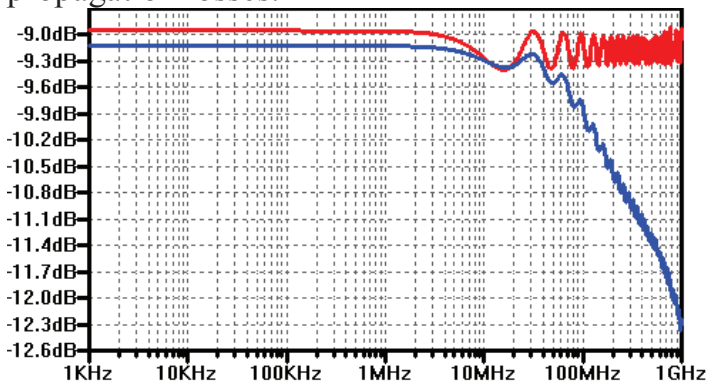

Figure 12. Sending $\left(\mathrm{V}_{\text {Dout } 1}\right)$ and receiving end voltages $\left(\mathrm{V}_{\mathrm{Din} 2}\right)$ as a function of frequency.
Figure 13 shows the near end crosstalk when a signal is sent on $\mathrm{D}_{\text {out1. The crosstalk is defined as }}$ $\mathrm{V}_{\text {Sin } 1} / \mathrm{V}_{\text {Dout1 }}$. This crosstalk is due solely to the connectors at either end as the shielded twisted pair model assumes no interaction between the differential mode and the other conductors.

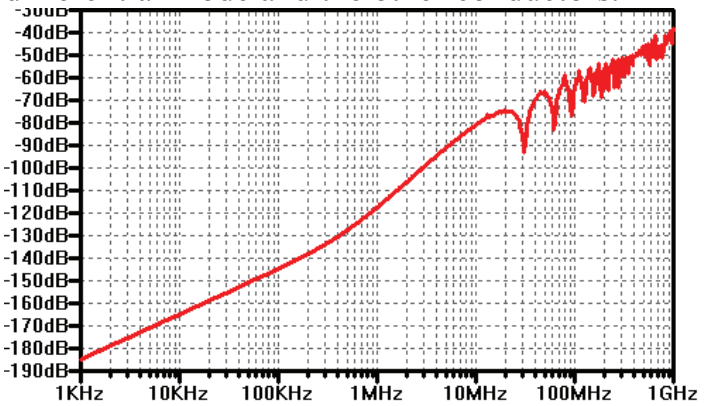

Figure 13. Near end crosstalk. $\mathrm{V}_{\sin 1} / \mathrm{V}_{\text {Dout1 }}$

\section{Conclusions}

Realistic models of multi-conductor cables have been developed for use within a Spice circuit simulation environment. These models include effects of interest in EMC/EMI studies i.e. transfer impedance coupling and incident field excitation. The models have been demonstrated in a range of scenarios of interest to the EMC community.

\section{Acknowledgments}

This work was carried out under the European Space Agency grant ESA 40000112765/14/NL/HK.

\section{References}

[1] Sacamos open source project website: www.sacamos.org

[2] C. R. Paul, "Analysis of Multi-conductor Transmission Lines",

Second edition, John Wiley \& Sons, 2008, ISBN 9780-470-13154-1.

[3] H.Xie, J.Wang, R.Fan, Y.Liu, "SPICE Models to Analyze Radiated and Conducted Susceptibilities of Shielded Coaxial Cables", IEEE Trans EMC, Vol 52, No 1, pp 215-222, 2010.

[4] C. R. Paul, "A SPICE model for multi-conductor transmission lines excited by an incident electromagnetic field," IEEE Trans EMC, vol36, no4, pp342-354, 1994.

[5] A. K. Agrawal, H. J. Price, "S. H. Gurbaxani, "Transient Response of Multi-conductor Transmission Lines Excited by a Nonuniform Electromagnetic Field," IEEE Trans EMC, vol 22, pp119-129, 1980.

[6] S. Greedy, C. Smartt, D. W. P. Thomas, "Open source cable models or EMI simulations," 2016 ESA Workshop on Aerospace EMC.

[7] CANBUS specification document ISO 118982:2003(E)

[8] Spacewire specification document ECSS-E-ST5012 
